\title{
APRESS: apical regulatory super system, serotonin, and dopamine interaction
}

Marty Hinz'

Alvin Stein ${ }^{2}$

Thomas Uncini ${ }^{3}$

'Clinical Research, NeuroResearch Clinics, Inc, Cape Coral, FL, USA;

${ }^{2}$ Stein Orthopedic Associates, Plantation, FL, USA; ${ }^{3}$ DBS Labs, Duluth, MN, USA
Correspondence: Marty Hinz 1008 Dolphin Drive, Cape Coral, FL 33904, USA

$\mathrm{Tel}+\mathrm{I} 2186262220$

Fax +I 2186261638

Email marty@hinzmd.com
This article was published in the following Dove Press journal:

Neuropsychiatric Disease and Treatment

2 September 201 I

Number of times this article has been viewed

Background: The monoamines serotonin and dopamine are known to exist in two separate states: the endogenous state and the competitive inhibition state. The presence of the competitive inhibition state has been known to science for many years, but from a functional standpoint it has been noted in the literature as being "meaningless."

Methods: A large database of monoamine transporter response to amino acid precursor administration variations with clinical outcomes was accumulated. In the process, a new organic cation transporter (OCT) model has been published, and OCT functional status determination along with amino acid precursor manipulation methods have been invented and refined.

Results: Methodology was developed whereby manipulation of the OCT, in the competitive inhibition state, is carried out in a predictable manner. This, in turn, has disproved the long-held assertion that the monoamine competitive inhibition state is functionally meaningless.

Conclusion: The most significant aspect of this paper is the documentation of newly recognized relationships between serotonin and dopamine. When transport of serotonin and dopamine are both in the competitive inhibition state, manipulation of the concentrations of one will lead to predictable changes in concentrations of the other. From a functional standpoint, processes regulated and controlled by changes to only serotonin can now be controlled by changes to dopamine, and vice versa, in a predictable manner.

Keywords: catecholamine, monoamine, competitive inhibition state

\section{Introduction}

Serotonin and the catecholamines (dopamine, norepinephrine, and epinephrine) belong to a group of chemicals herein known as "monoamines." The monoamines function independently, controlling and/or regulating bodily functions. These functions include, but are not limited to, neurotransmitter, neurohormone, regulatory, autocrine, paracrine, and autonomic control. ${ }^{1-3}$

This paper documents novel observations of the competitive inhibition state, which was previously thought to be functionally meaningless. The physiologic observations of this state are deemed the "apical regulatory super system" (APRESS), which occurs with simultaneous administration of serotonin and dopamine amino acid precursors in significant amounts.

"Super system" is defined as the fusion of two independent systems into one. Changes to one or more components of either system affect changes to all components in a predictable manner. In the balanced competitive inhibition state, ${ }^{3-8}$ serotonin and catecholamines undergo super system fusion. 
Monoamines exist in one of three states: endogenous, unbalanced competitive inhibition, and balanced competitive inhibition..$^{3-8}$ The "endogenous state" is achieved by dietary intake alone when no supplemental monoamine amino acid precursors are being administered. ${ }^{3-8}$

The competitive inhibition state cannot be achieved through dietary modification; it is established when significant amounts of monoamine precursors are simultaneously administered. ${ }^{3-8}$ Competitive inhibition state literature notes “... functional relevance of the competitive inhibitory effect ... is most probably meaningless." ${ }^{\prime 9}$ Functional relevance is achieved with the ability to assay and regulate transport of the monoamines in a predictable manner with organic cation transporter (OCT) functional status determination. Except by random chance, it is impossible to achieve the balanced competitive inhibition state without OCT functional determination..$^{3-8}$ Differentiating "unbalanced" and "balanced" competitive inhibition is discussed later in this paper.

The foundation of APRESS is that in the competitive inhibition state, transport changes to one monoamine lead to predictable changes in all monoamines. These changes are not intuitive. Endogenous state observations are not applicable to APRESS (see Tables 1-4). This writing introduces APRESS as a novel physiologic state adhering to unique transporter properties that are counterintuitive to rules of the endogenous state. This paper discusses only the following limited aspects of monoamine interaction in APRESS.

- Functions impacted and/or controlled in the endogenous state only by changes in serotonin concentrations may also be impacted or controlled by changes in dopamine concentrations in APRESS.

- Functions impacted and/or controlled in the endogenous state only by changes in dopamine concentrations may also be impacted or controlled by changes in serotonin concentrations in APRESS.

\section{Methods and materials}

Since 2009, the authors of this paper have published eleven peer-reviewed original research papers relating to the simultaneous manipulation of the serotonin and catecholamine systems with amino acid precursors under guidance of OCT assay interpretation..$^{3-8,10-14}$ APRESS embodies the common thread of these writings: serotonin and catecholamine fusion into one system. Previous publications outlined much of the novel scientific foundation of APRESS, but its impact, novel abilities and other considerations have not been fully explored and documented.

Following are research components used to define the novel physiologic attributes of APRESS in the body. These components, observations, and networking applications include, but are not limited to:

- Over 1000 monoamine databases relating to the endogenous and competitive inhibition states. ${ }^{15}$

- A master database documenting over 2 million patient-days of monoamine amino acid transporter manipulation. ${ }^{15}$

- Review and interpretation of over 100,000 urinary monoamine assays in the endogenous or competitive inhibition state. ${ }^{15}$

- Defining the three-phase monoamine transporter response of serotonin and dopamine observed during simultaneous administration of their precursors..$^{3-8,10-14}$

- A network of over 1000 physicians manipulating nutrients in APRESS. ${ }^{15}$

Serotonin and dopamine filtered at the glomerulous are metabolized by the kidneys; significant amounts do not make it into the final urine. Newly synthesized renal serotonin and dopamine meet one of two fates, as illustrated in Figure 1. The three-phase response of urinary monoamines is used to determine the functional status of the basolateral OCT2 of the proximal convoluted renal tubule cells. ${ }^{3-8,10-14}$ Urinary levels are dependent upon the interaction of the basolateral OCT2 and the apical OCTN2 in transporting newly synthesized monoamines out of the proximal convoluted renal tubule cells (see Figure 1)..$^{3-8,11,12,16}$

Proper OCT interpretation requires obtaining two or more urinary monoamine assays while taking significant amounts of varied precursor dosing values consistently for 5 days minimum to achieve equilibrium. Serial assays are then compared to determine the impact of precursor dosing value changes..$^{3-8,10-14}$

The following urinary monoamine values were reported in $\mu \mathrm{g}$ of monoamine per $\mathrm{g}$ of creatinine to compensate for specific gravity fluctuations. A urinary serotonin or dopamine

Table I Impact of increasing amino acid precursor dosing values of System A in phase 3

\begin{tabular}{lllllll}
\hline & Amino acid intake & Synthesis & Metabolism & Transport & Post transporter & Urinary \\
\hline System A & Increased & Increased & Increased & Increased & Increased & Increased \\
System B & Same & Decreased & Increased & Decreased & Decreased & Increased \\
\hline
\end{tabular}

Note: The response to increasing one monoamine precursor of serotonin or dopamine in competitive inhibition. 
Table 2 Impact of decreasing amino acid precursor dosing values of System A in phase 3

\begin{tabular}{lllllll}
\hline & Amino acid intake & Synthesis & Metabolism & Transport & Post transporter & Urinary \\
\hline System A & Decreased & Decreased & Decreased & Decreased & Decreased & Decreased \\
System B & Same & Increased & Decreased & Increased & Increased & Decreased \\
\hline
\end{tabular}

Note: The response to decreasing one monoamine precursor of serotonin or dopamine in competitive inhibition.

value less than 80 or 475 , respectively, is defined as a phase 2 response. A urinary serotonin or dopamine value greater than 80 or 475 , respectively, is interpreted as being in phase 1 or phase 3. If a direct relationship is found between amino acid dosing and urinary assay response, it is a phase 3 response. An inverse correlation is a phase 1 response. The phase 3 therapeutic range for urinary serotonin is $80-240$. The phase 3 therapeutic range for urinary dopamine is $475-1100 .^{3-8,10-14}$

Urine samples were collected 6 hours prior to bedtime after a minimum of 1 week on a specific dosing value, with no missed doses of amino acid precursors. The most frequent collection time point was 4 PM. Samples were stabilized in $6 \mathrm{~N} \mathrm{HCl}$ to preserve the monoamines and shipped to DBS Laboratories (Duluth, MN) which is operated under the direction of one of the authors (Thomas Uncini, MD). Urinary monoamines were assayed utilizing commercially available radioimmunoassay kits (3 CAT RIA IB88501 and IB89527, both from Immuno Biological Laboratories, Inc, Minneapolis, MN). The DBS laboratory is accredited as a high complexity laboratory by Clinical Laboratory Improvement Amendments. OCT assay interpretation was performed by one of the authors (Marty Hinz). ${ }^{3-8,10-14}$

\section{Results}

Three primary functions affect intracellular and extracellular serotonin and catecholamine levels: synthesis, metabolism, and transport. ${ }^{3-5,7,8,10-12}$ These functions occur in either the endogenous or competitive inhibition state..$^{3-8}$

The relationship between serotonin and dopamine regarding synthesis, metabolism, and transport in the endogenous state appears to be random. Matched pairs $t$-test analysis of endogenous urinary monoamines reveals significant day-to-day changes $(P<0.05)$ in a subject. In the competitive

Table 3 Urinary monoamine concentrations per phase

\begin{tabular}{lll}
\hline & Serotonin & Dopamine \\
\hline Phase I & $>80$ & $>475$ \\
Phase 2 & $<80$ & $<475$ \\
Phase 3 & $>80$ & $>475$ \\
\multicolumn{2}{l}{ Phase 3 therapeutic ranges } & \\
Serotonin & Dopamine & \\
$80-240$ & $475-1100$ & \\
\hline
\end{tabular}

Note: Units are in $\mu g$ of monoamine per g creatinine. inhibition state, predictable changes occur to all monoamine components with changes to individual precursor dosing values of either system. ${ }^{6,10,12}$

Aromatic L-amino acid decarboxylase (AAAD) catalyzes serotonin and dopamine synthesis. Monoamine oxidase (MAO) catalyzes metabolism of serotonin and dopamine. OCT transports monoamines and their precursors in and out of proximal convoluted renal tubule cells. When significant amounts of balanced serotonin and dopamine precursors are administered simultaneously under guidance of OCT assay optimization, the balanced competitive inhibition of APRESS is established. ${ }^{6,10,12}$

Immediate precursors of serotonin and dopamine presenting at the AAAD need to be in balance. If not, precursors of one system will dominate AAAD, compromising nondominant monoamine synthesis. ${ }^{6,10,11}$

Serotonin and dopamine need to be in balance or the dominant monoamine increases MAO activity and metabolism of the nondominant system. ${ }^{6,10,12}$

Monoamine renal physiology is complex. Prior to the authors writing this paper, the sequence of events from the monoamines and their precursors being filtered at the glomerulous to them appearing in the system or final urine had not been documented.

Until this research, direct clinical measurement and evaluation of monoamine OCT functional status did not exist, rendering the competitive inhibition state functionally meaningless. ${ }^{9}$

Serotonin, dopamine, and their precursors filtered at the glomerulous are taken up from the proximal tubules by OCT2 transporters into the proximal convoluted renal tubule cells where the monoamines are metabolized. Significant amounts do not make it to the final urine under normal conditions..$^{3-8,11,12}$ Precursors are then synthesized into new monoamines, which are preferentially transported by the basolateral OCT2 to the system or excreted via the apical OCTN2 as urinary waste. Interpretation of urinary monoamine levels in the competitive inhibition state is an interpretation of the functional status of the basolateral OCT2. . $^{3,11,12}$

Previous writings by the authors of this paper refined the basolateral OCT2 transporter model. ${ }^{3}$ The OCT of the liver, kidneys, bowels, and brain are "identical and 
Table 4 Effect of increasing only L-dopa dosing values in the competitive inhibition state

\begin{tabular}{lllllll}
\hline Day \# & $\begin{array}{l}\text { Urinary } \\
\text { serotonin }\end{array}$ & $\begin{array}{l}\text { Urinary } \\
\text { dopamine }\end{array}$ & $\begin{array}{l}\text { Serotonin } \\
\text { phase }\end{array}$ & $\begin{array}{l}\text { Dopamine } \\
\text { phase }\end{array}$ & $\begin{array}{l}\text { 5-HTP mg/day } \\
\text { dosing value }\end{array}$ & $\begin{array}{l}\text { L-dopa mg/day } \\
\text { dosing value }\end{array}$ \\
\hline 0 (test I) & 84 & 289 & 3 & 2 & 900 & 120 \\
8 (test 2) & 473 & 786 & 3 & 3 & 900 & 360 \\
\hline
\end{tabular}

Notes: Serotonin phase 3/3; Dopamine phase $2 / 3$; units are in $\mu g$ of monoamine per $g$ creatinine.

Abbreviation: HTP, hydroxytryptophan.

homologous." Precursors cross the blood-brain barrier then come to equilibrium throughout the body. ${ }^{16}$ Under the dual gate lumen transporter model, there are separate serotonin and dopamine gates at the lumen entrance. These gates independently regulate lumen access by the respective monoamine. While either gate can be partially closed, blocking access, both gates are never simultaneously partially closed. ${ }^{3}$

Serotonin and dopamine basolateral OCT2 transport exists in three transport phases. In phase 1 the entrance gate is partially closed, restricting access to the nonsaturated lumen. If the gate at the lumen is partially closed (phase 1), it will open as the total amount of serotonin and dopamine presenting at the transporter increases. In phase 2 the gate of the nonsaturated lumen is open. In phase 3 the entrance gate of the saturated lumen is open..$^{3-8,10-14}$

Now the question becomes: how can functions controlled only by serotonin or dopamine in the endogenous state be controlled in a predictable manner by changes to either in the competitive inhibition state?

Once the transporter is saturated with both serotonin and dopamine (phase 3), an increase in one monoamine being transported will cause the amount of the other monoamine being transported to decrease. Tables 1 and 2 document the

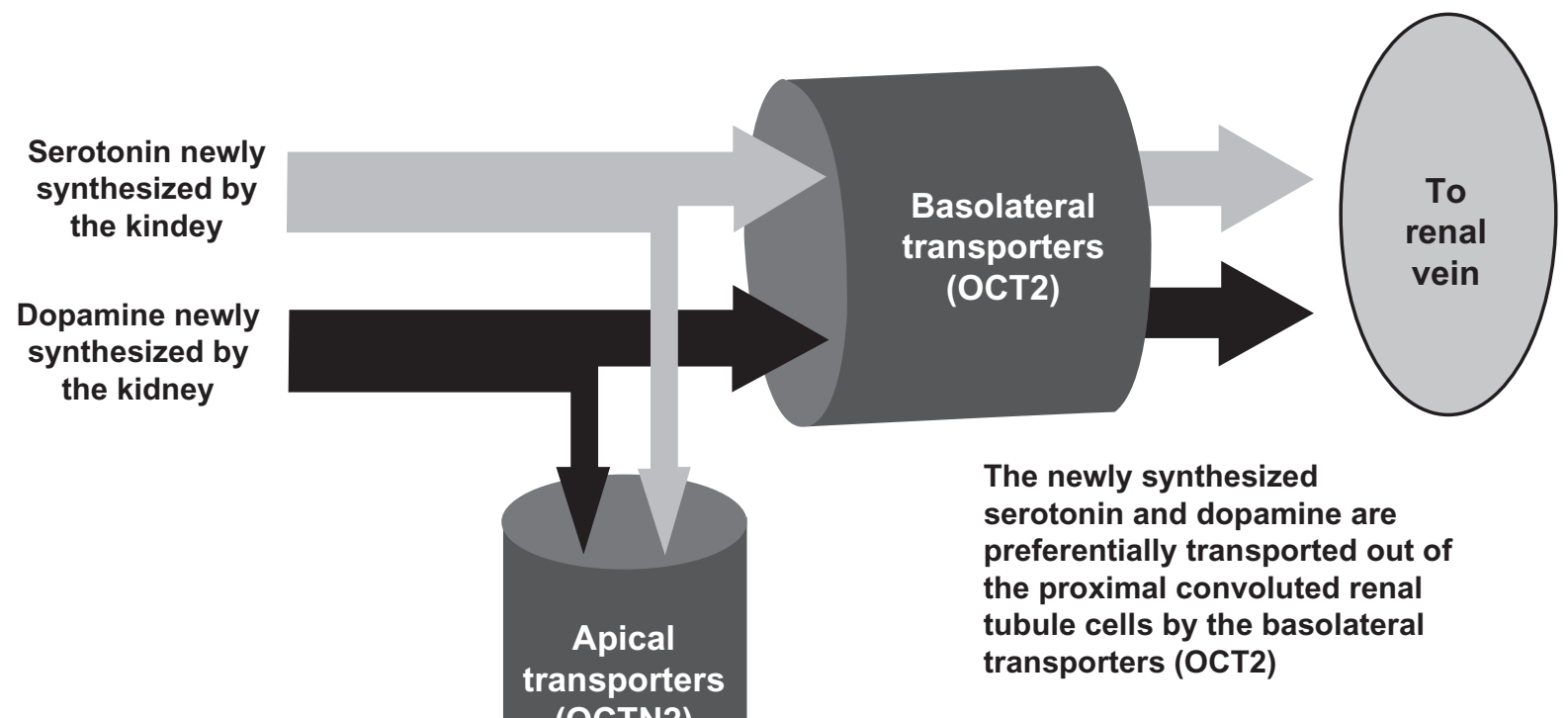

The serotonin and dopamine not transported by the basolateral transporter are transported as waste to the final urine via the apical transporters (OCTN2)

\section{To final urine}

Figure I The interaction of OCT2 and OCTN2. Newly synthesized serotonin and dopamine are transported preferentially by the basolateral OCT2. Functional status of the OCT2 is determined by assaying the urinary monoamines not transported by the OCT2, which are transported by the OCTN2 to the final urine as waste. ${ }^{3-8,11}$ Abbreviation: OCT, organic cation transporter. 
impact of placing both systems into competitive inhibition as verified by OCT assay determination..$^{3-8,10-14}$

In Tables 1 and 2, "System A" is either L-dopa or 5-hydroxytryptophan (immediate dopamine and serotonin precursors respectively), with "System B" the opposite precursor. With both systems in phase 3, a single precursor increase leads to a decrease in the post-transporter levels of the other system through competitive inhibition, just as a decrease of one amino acid precursor leads to an increase in post-transporter levels of the other system. In the competitive inhibition state, these levels can be changed by making changes to amino acid precursor dosing values of either system.

Interpretation of urinary levels of serotonin and dopamine, with amino acid dosing changes in the competitive inhibition state, are complex. In Table 1, the precursor increase causes decreased synthesis of the nondominant monoamine (system B) through AAAD competitive inhibition. Increased activity of the MAO enzyme system is induced, leading to increased metabolism of the nondominant monoamine.

The key to optimal balanced control is to place the serotonin and dopamine in the therapeutic phase 3 ranges of Table 3. This cannot be done empirically. The dosing levels of 5-hydroxytryptophan (5-HTP) need to reach the phase 2/phase 3 inflection point between 37.5 and $2400 \mathrm{mg}$ per day. ${ }^{15}$ The L-dopa dosing level to reach this inflection point is between 30 and $2100 \mathrm{mg}$ per day. Dosing values of serotonin precursors are independent from dopamine precursors, meaning any combination of precursors in a large spectrum is possible. OCT assay to determine levels of serotonin and dopamine not transported by the basolateral OCT2 is required. ${ }^{3-8,10-14}$

In test 1 of Table 4 , serotonin is phase 3 and dopamine transport is phase 2. Between tests, L-dopa was increased to $360 \mathrm{mg}$ per day, causing an increase in dopamine transported by the OCT2 and in the urine. Less serotonin was then transported by the OCT2 as dopamine transport increased, excluding serotonin from the OCT2 transporter through competitive inhibition, and more serotonin appeared in the final urine as waste. In the first assay, serotonin was $84 \mu \mathrm{g}$ of serotonin per $g$ creatinine and in phase 3 . An increase in L-dopa leads to a decrease in the serotonin phase 2 /phase 3 inflection point (inverse relationship). The same is true regarding 5-HTP increases and the dopamine phase 2 /phase 3 inflection point.

In test 2, APRESS effect is not optimal. The urinary serotonin is too high, causing excessive exclusion of dopamine transport even though the dopamine is in the therapeutic range. Optimal serotonin and dopamine transport is seen only with both serotonin and dopamine in their phase 3 therapeutic ranges. Even though on test 2 the urinary dopamine is in the phase 3 therapeutic range, this observation is meaningless until both serotonin and dopamine are simultaneously in therapeutic ranges. If both systems are not in therapeutic phase 3 ranges defined in Table 3, further optimization is needed to maximize transporter balance. With the lowering of daily 5-HTP dosing values due to OCT2 transporter imbalance, the urinary dopamine levels at the OCTN2 transporter entrance will drop secondary to decreased serotonin transport, facilitating increased dopamine transport. This causes less serotonin and dopamine to show up in the final urine as waste. The general direction of urinary serotonin levels in transport and final urinary levels can be predicted in the competitive inhibition state; the exact amount of movement secondary to amino acid precursor changes, however, is individualized..$^{3-8,10-14}$

Full interpretation of large-scale results may be quite confusing at times for the uninitiated. Extraordinarily complex relationships not covered in this writing exist based on determination of OCT2 transporter status. These complexities are beyond the scope of this paper..$^{3-8,10-14}$

\section{The unbalanced competitive inhibition state, a pathologic state}

The hallmark of the unbalanced competitive inhibition state is "pathological depletion of monoamine components." When unbalanced amino acid precursors are administered, one system dominates and the other is nondominant. The dominant system overwhelms and depletes the nondominant system. Most significant depletions occur in weeks or months although some may take years. ${ }^{4,5,8,10,11}$

- L-tryptophan or 5-HTP depletes dopamine when dominant. ${ }^{4,5,8,10,11}$

- L-tyrosine or L-dopa depletes serotonin when dominant. ${ }^{4,5,8,10,11}$

L-dopa and 5-HTP are the immediate dopamine and serotonin precursors, respectively. Both are synthesized into the monoamine without biochemical feedback regulation. ${ }^{7}$ The following clinical situation illustrates unbalanced depletion of a nondominant system.

In Parkinson's disease, serotonin and dopamine are depleted by the disease. L-dopa treatment alone leads to serotonin depletion by inhibiting serotonin AAAD synthesis. Administering only L-dopa increases the activity of the MAO system, depleting the nondominant serotonin when balanced levels of 5-HTP are not being administered. Side effects associated with administration of only L-dopa, which are 
related to serotonin imbalance, include but are not limited to tachyphylaxis due to serotonin depletion, problems of improperly balanced monoamines, problems caused by dopamine fluctuations, and problems resulting from sulfur amino acid depletion. ${ }^{7}$

Problems associated with dominant levels of 5-HTP are a mirror image of the L-dopa problems. The common practice of administering only 5-HTP without properly balanced dopamine precursors depletes dopamine. ${ }^{7}$

\section{Discussion}

Functions dependent on only serotonin or dopamine concentrations in the endogenous state can be impacted in a predictable manner by changes in levels of either in the balanced competitive inhibition state which is the foundation of APRESS. ${ }^{4,5,8,10,11}$ APRESS physiology is not intuitive. An intimate knowledge of monoamine physiology in the endogenous state may even be a distraction to mastering the complex state of APRESS. Observations and rules formulated for the endogenous and unbalanced competitive inhibition states are typically not valid in guiding amino acid precursor adjustments. . $^{3-8,10-14}$

The physiological and clinical management observations entered into the APRESS catalog have just started. It contains the following:

- Functions controlled by dopamine in the endogenous state can be controlled with changes to serotonin and vice versa.

- The balanced approach does not deplete monoamines..$^{3-8,10-14}$

- Administration of targeted and balanced amino acid precursors leads to optimal results in disease and dysfunction management. ${ }^{3-8,10-14}$

- The approach directly addresses and treats the cause of problems, unlike the symptom management approach with prescription drugs. ${ }^{3-8,10-14}$

- Properly balanced serotonin and dopamine does away with side effect dosing value barriers providing the opportunity to reach levels of needed amino acid intake not possible in the unbalanced state..$^{3-8,10-14}$

- Parkinson's disease patients can be treated with L-dopa levels needed to control symptoms without reaching a side-effect induced dosing barrier. ${ }^{7}$

- Management of the extensive list of side effects associated with L-dopa and carbidopa in treatment of Parkinson disease. ${ }^{7}$

- Bipolar disorder cycling on the depressive pole can be differentiated from major affective disorder then treated effectively. ${ }^{11}$
- Refractory depression can be successfully treated without drug side effects.

- In Crohn's disease, OCT genetic transporter defects that cause Crohn's symptoms and interstitial colonic serotonin to be markedly elevated can be treated. ${ }^{4}$

- Attention deficit hyperactivity disorder preliminary treatment studies $(\mathrm{N}=85)$ published in 2011 reveal efficacy results greater than atomoxetine or methylphenidate. ${ }^{5}$

\section{Conclusion}

Serotonin and dopamine may exist in three basic states: the endogenous state, the unbalanced competitive inhibition state, and the balanced competitive inhibition state. Physiologic observations in the endogenous state have no bearing or relationship with the competitive inhibition state.

The balanced competitive inhibition state is the foundation for APRESS. APRESS is a registry of the activities impacted by manipulating the balanced competitive inhibition state. The ability to affect and regulate bilateral serotonin or dopamine concentration changes with a single monoamine adjustment in a predictable manner is the foundation of APRESS. This leads to the ability to define interactions and gain control in a physiologic state that until now was thought to be functionally meaningless.

APRESS is indeed based on a unique physiologic state that is not meaningless. It is a new frontier that cannot be achieved through dietary manipulation alone or without OCT functional status determination, a procedure that can be performed in medical clinics.

APRESS is not a state that can be optimally achieved by empirical administration of amino acid precursors. Once in this clinical competitive inhibition state, the scientific observations of the endogenous state are no longer valid. APRESS is a new physiological world in and of itself. It is in need of research to define its far-reaching parameters and implications. It is the goal of this writing to stimulate interest and discussion relating to this novel state known as APRESS.

\section{Disclosure}

MH owns NeuroResearch Clinics, Inc; AS reports no disclosures; TU reports a directorship of DBS Laboratories.

\section{References}

1. Pazos-Moura C, Ortiga-Carvalho T, Gaspar de Moura E. The autocrine/ paracrine regulation of thyrotropin secretion. Thyroid. 2004;13:2.

2. O'Hara J, Ho W, Linden D, et al. Enteroendocrine cells and 5-HT availability are altered in mucosa of guinea pigs with TNBS ileitis. Am J Physiol Gastrointest Liver Physiol. 2004;287:G998-G1007.

3. Hinz M, Stein A, Uncini T. The dual-gate lumen model of renal monoamine transport. Neuropsychiatr Dis Treat. 2010;6:387-392. 
4. Stein A, Hinz M, Uncini T. Amino acid-responsive Crohn's disease: a case study. Clin Exp Gastroenterol. 2010;3:171-177.

5. Hinz M, Stein A, Neff R, et al. Treatment of attention deficit hyperactivity disorder with monoamine amino acid precursors and organic cation transporter assay interpretation. Neuropsychiatr Dis Treat. 2011;7:31-38.

6. Hinz M, Stein A, Uncini T. Urinary neurotransmitter testing: considerations of spot baseline norepinephrine and epinephrine. Open Access J Urol. 2011;3:19-24.

7. Hinz M, Stein A, Uncini T. Amino acid management of Parkinson's disease: a case study. Int J Gen Med. 2011;4;165-174.

8. Hinz M. Depression. In: Kohlstadt I, editor. Food and Nutrients in Disease Management. Boca Raton, FL: CRC Press; 465-481.

9. Soares-da-Silva P, Pinto-do-O PC. Antagonistic actions of renal dopamine and 5-hydroxytryptamine: effects of amine precursors on the cell inward transfer and decarboxylation. Br J Pharmacol. 1996; 117:1187-1192.

10. Hinz M, Stein A, Trachte G, Uncini T. Neurotransmitter testing of the urine: a comprehensive analysis. Open Access J Urol. 2010;2: $177-183$.
11. Hinz M, Stein A, Uncini T. A pilot study differentiating recurrent major depression from bipolar disorder cycling on the depressive pole. Neuropsychiatr Dis Treat. 2010;6:741-747.

12. Hinz M, Stein A, Uncini T. "Non-validity and clinical relevance of neurotransmitter testing": a review of the paper. Funct Neurol Rehabil Ergon. 2011. In press.

13. Trachte G, Uncini T, Hinz M. Both stimulatory and inhibitory effects of dietary 5-hydroxytryptophan and tyrosine are found on urinary excretion of serotonin and dopamine in a large human population. Neuropsychiatr Dis Treat. 2009;5:227-235.

14. Hinz M, Stein A, Uncini T. The validity of urinary monoamine assay sales under the "spot baseline urinary neurotransmitter testing marketing model.” Int J Nephrol Renovascular Dis. 2011;4:101-113.

15. NeuroResearch Clinics, Inc. Medical research, medical education databases and records. 1008 Dolphin Dr, Cape Coral, FL 33904, USA.

16. Koepsell H. Organic cation transporters in the intestine, kidney, liver, and brain. Аnпu Rev Physiol. 1998;60:243-266.
Neuropsychiatric Disease and Treatment

\section{Publish your work in this journal}

Neuropsychiatric Disease and Treatment is an international, peerreviewed journal of clinical therapeutics and pharmacology focusing on concise rapid reporting of clinical or pre-clinical studies on a range of neuropsychiatric and neurological disorders. This journal is indexed on PubMed Central, the 'PsycINFO' database and CAS, and is the official

\section{Dovepress}

journal of The International Neuropsychiatric Association (INA). The manuscript management system is completely online and includes a very quick and fair peer-review system, which is all easy to use. Visit http://www.dovepress.com/testimonials.php to read real quotes from published authors.

Submit your manuscript here: http://www.dovepress.com/neuropsychiatric-disease-and-treatment-journal 